\title{
Anticoagulation in COVID-19: Effect of Enoxaparin, Heparin, and Apixaban on Mortality
}

\author{
Henny H. Billett ${ }^{1, *}$ Morayma Reyes-Gil ${ }^{2, *}$ James Szymanski ${ }^{20} \quad$ Kenji Ikemura $^{2} \quad$ Lindsay R. Stahl ${ }^{3}$ \\ Yungtai Lo ${ }^{4}$ Shafia Rahman ${ }^{1}$ Jesus D. Gonzalez-Lugo ${ }^{1}$ Margarita Kushnir ${ }^{1}$ Mohammad Barouqa ${ }^{2}$ \\ Ladan Golestaneh ${ }^{5}$ Eran Bellin ${ }^{4, *}$
}
${ }^{1}$ Division of Hematology, Departments of Oncology and Medicine, Montefiore Medical Center, Albert Einstein College of Medicine, Bronx, New York, United States
2 Department of Pathology, Montefiore Medical Center, Albert Einstein College of Medicine, Bronx, New York, United States
${ }^{3}$ Montefiore Information Technology, Montefiore Medical Center, Albert Einstein College of Medicine, Bronx, New York, United States
${ }^{4}$ Department of Epidemiology and Population Health and Medicine, Montefiore Medical Center, Albert Einstein College of Medicine, Bronx, New York, United States
${ }^{5}$ Division of Nephrology, Montefiore Medical Center, Albert Einstein College of Medicine, Bronx, New York, United States

\begin{abstract}
Address for correspondence Henny H. Billett, MD, Division of Hematology, Departments of Oncology/Medicine, Montefiore Medical Center, Albert Einstein College of Medicine, 3411 Wayne Avenue, Bronx, NY 10467, United States (e-mail: hbillett@montefiore.org).
\end{abstract}

Thromb Haemost 2020;120:1691-1699.

\begin{abstract}
Keywords

- anticoagulation

- apixaban

- COVID-19

- D-dimer

- enoxaparin

- mortality

Background Mortality in coronavirus disease of 2019 (COVID-19) is associated with increases in prothrombotic parameters, particularly D-dimer levels. Anticoagulation has been proposed as therapy to decrease mortality, often adjusted for illness severity. Objective We wanted to investigate whether anticoagulation improves survival in COVID-19 and if this improvement in survival is associated with disease severity.

Methods This is a cohort study simulating an intention-to-treat clinical trial, by analyzing the effect on mortality of anticoagulation therapy chosen in the first 48 hours of hospitalization. We analyzed 3,625 COVID-19+ inpatients, controlling for age, gender, glomerular filtration rate, oxygen saturation, ventilation requirement, intensive care unit admission, and time period, all determined during the first 48 hours.

Results Adjusted logistic regression analyses demonstrated a significant decrease in mortality with prophylactic use of apixaban (odds ratio [OR $0.46, p=0.001$ ) and enoxaparin $(\mathrm{OR}=0.49, p=0.001)$. Therapeutic apixaban was also associated with decreased mortality (OR $0.57, p=0.006$ ) but was not more beneficial than prophylactic use when analyzed over the entire cohort or within D-dimer stratified categories. Higher D-dimer levels were associated with increased mortality $(p<0.0001)$. When adjusted for these same comorbidities within D-dimer strata, patients with D-dimer levels $<1 \mu \mathrm{g} / \mathrm{mL}$ did not appear to benefit from anticoagulation while patients with D-dimer levels $>10 \mu \mathrm{g} / \mathrm{mL}$ derived the most benefit. There was no increase in transfusion requirement with any of the anticoagulants used.

Conclusion We conclude that COVID-19+ patients with moderate or severe illness benefit from anticoagulation and that apixaban has similar efficacy to enoxaparin in decreasing mortality in this disease.
\end{abstract}

\footnotetext{
* Equal authorship.
}

received

August 25, 2020

accepted after revision

September 24, 2020 (c) 2020. Thieme. All rights reserved. Georg Thieme Verlag KG,

Rüdigerstraße 14,

70469 Stuttgart, Germany
DOI https://doi.org/

10.1055/s-0040-1720978.

ISSN 0340-6245. 


\section{Introduction}

Coronavirus disease of 2019 (COVID-19) is a heterogeneous disease, ranging from asymptomatic illness in some to rapid death in others. Trying to understand the manifestations of the disease, and whether these could be used both prognostically and therapeutically, has been a focus of intense work throughout the world. The Chinese were the first to report a COVID-19 coagulopathy, particularly among patients with severe forms of the illness. ${ }^{1}$ There are now data that coagulation parameters, particularly D-dimer levels, are prognostically significant for mortality in COVID-19 and that patients with COVID-19 are at increased risk of both arterial and venous thrombosis. ${ }^{2-4}$

There has been a suggestion that anticoagulation (AC) may be of benefit in these patients, either in reduced venous thromboembolism rate or mortality, although the evidence is sparse and not all experts agree. ${ }^{5,6}$ In Wuhan, 449 patients with severe COVID-19 were retrospectively analyzed regarding the potential benefit of AC. ${ }^{7}$ All of these patients received antiviral and supportive therapy in addition to $A C$ and all $A C$ was grouped, prophylactic and therapeutic. Although the study was done at the early onset of COVID-19 pandemic and did not analyze for other variables, this was an early indication that AC might affect mortality. A similar beneficial outcome for $A C$ was seen in a smaller retrospective analysis in New York grouping all types of anticoagulants. ${ }^{8,9}$

We investigated the relationship of both type and intensity of AC to mortality and whether objective parameters like D-dimer levels might differentiate outcomes with specific AC regimens.

\section{Methods}

An optional AC algorithm for hospitalized COVID-19+ patients was initiated as best clinical judgment on April 4, 2020 and provided to all physicians. This included specific recommendations for surveillance monitoring of D-dimer levels as well as recommendations for prophylactic or therapeutic AC with either indirect or direct oral anticoagulants (DOACs). This is a cohort study of these patients hospitalized from March 1 to May 30, 2020 during the COVID-19 pandemic.

\section{Study Population}

All patients admitted to one of three Montefiore Medical Center Hospitals in the Bronx from March 1 to April 26, 2020 and who tested positive for COVID-19 for the first time within 24 hours of admission were eligible for inclusion. These patients were followed until May 30, 2020.

\section{Data Collection}

After obtaining Institutional Review Board (IRB) approval from Albert Einstein College of Medicine, we queried Clinical Looking Glass, ${ }^{10,11}$ a user-friendly cohort building and outcome analysis software application with access to the electronic medical record data, for all positive COVID-19 tests performed by Montefiore Health System between March 1, 2020 to April 26, 2020.

\section{Cohort Creation and Outcome Analysis}

Using observational data retrospectively collected, we imitated a clinical trial by collecting baseline variables in the first 48 hours of the admission, analytically assigned patients surviving that interval to anticoagulant group based upon their last anticoagulant order in the first 48 hours, and then followed them from the 48th hour to outcome of death or live discharge. Those not discharged from the hospital by May 30 , 2020 or participating in an IRB-approved experimental clinical trial were excluded. If in the first 48 hours the last anticoagulant order did not have a duration beyond a single dose the patient was assigned to the category of no AC. This assignment is an imitation of the intention-to-treat methodology used in randomized controlled trials.

Data collected included age, gender, body mass index (BMI), estimated glomerular filtration rate (eGFR), oxygen saturation as determined by pulse oximetry, vital signs, ventilator requirement, intensive care unit (ICU) admission, D-dimer level, and choice of AC within the first 48 hours of presentation. D-dimer level was categorized as $<1,1$ to $<3$, $\geq 3$ to 10 , and $\geq 10 \mu \mathrm{g} / \mathrm{mL}$; eGFR was categorized as 0 to 15 , 15 to 30,30 to 60 , and $\geq 60 \mathrm{~mL} / \mathrm{min}$. The highest two eGFR categories were collapsed during analysis.

Physician intent to treat with a specific AC medication and style was thus established in the first 48 hours of admission before outcome was sought. Logistic regression models for outcome were built adjusting for covariates that coincided with intent and preceded the search for outcome.

We classified AC regimen intensity as therapeutic or prophylactic and noted route as shown in -Supplementary Table S1 (available in the online version). All dosages were reviewed individually, with particular attention to GFR, age, and weight, to ensure correct designation. All intravenous unfractionated heparin (UFH) was deemed to have been given for therapeutic intent; anti- $\mathrm{X}_{\mathrm{a}}$ levels were not monitored and partial thromboplastin time data were not considered reliable within the COVID-19 "coagulopathy." Patients on chronic AC were excluded, as were patients on other clinical trials. Cohorts were created recognizing four distinct time convenience periods in the COVID-19 influx of patient admissions: March 1 to 31, April 1 to 11, April 12 to 26, and April 27 to May 30 and controlled for these time periods.

\section{Statistical Method}

Stata 16 (Stata Press, College Station, Texas, United States) was used for all statistical analyses. Univariate survival analysis was performed with the Kaplan-Meier statistic and $\log$ rank test. Multivariate logistic regression modeling was performed on discharged patients (dead or alive) by May 30. Patients who were still hospitalized by May 30 were eliminated from consideration.

\section{Results}

\section{Study Population}

A total of 4,234 patients who were found to be COVID-19 positive for the first time within 24 hours of admission were evaluated. 252 patients were participating in randomized 
clinical therapy trials and were excluded, leaving 3,982 potentially evaluable patients for AC intent. A further 175 patients were eliminated due to lack of sufficient data or were under 18years of age, leaving 3,807 patients. Note that 182 patients were further eliminated because they were not discharged by study termination (22) or had died within the first 48 hours (160). This left 3,625 patients as patients evaluable for the study (-Supplementary Fig. $\mathbf{~} \mathbf{1}$, available in the online version), of which 2,450 had complete data sets. Demographics and initial 48-hour descriptors are summarized in $\boldsymbol{- T a b l e ~} \mathbf{1}$.

\section{Adjustments for Time}

Adjustments were made for four time periods. The first corresponded to the initial wave of 21 days (March 11-31) with 759 patients or 36.1 COVID-19+ patients/day; time period 2 (April 1-11) was one of maximum surge and had 1,666 COVID-19 admissions in 11 days or 151.5 COVID-19+ patients/day; period 3 (April 12-26) is where the "curve flattening" started to occur and 797 COVID-19+ patients were admitted in 15 days or 53.1 patients/day. Period 4 (April 27-May 30) was when the admission rate decreased markedly (369 patients in 33 days or 11.2 patients/day), expertise was greater, and beds and supplies were sufficient. It was during the initial surge that an $A C$ protocol (-Supplementary Fig. S2, available in the online version) was developed which stratified therapy according to D-dimer level. Survival in the third time period increased significantly (-Fig. 1). Since the later time periods ( 3 and 4 ) were associated with significantly decreased mortality that may or may not have been associated with choice of anticoagulant therapy, logistic models were adjusted for time "wave."

\section{Therapy Choice}

Despite the protocol, at 48 hours, no orders for AC had been given for $17.6 \%$ of the admitted patients; $58.8 \%$ received standard thromboprophylaxis, $4.0 \%$ received high dose prophylaxis, and 19.6\% received therapeutic AC. For those who chose prophylaxis, UFH given subcutaneously either at the standard twice-daily dose or the high, three times daily dose was preferred for prophylaxis $(42.7 \%)$ while apixaban was the overall preferred therapeutic regimen (66.8\%). For patients with D-dimer values $<3 \mu \mathrm{g} / \mathrm{mL}$ for whom thromboprophylaxis had been recommended by protocol, $13.2 \%$ received full therapeutic doses and $12.3 \%$ were not prescribed any AC. Similarly, for those patients with D-dimer levels $\geq 3 \mu \mathrm{g} / \mathrm{mL}$ for whom therapeutic dose had been recommended by protocol, $16.0 \%$ received thromboprophylaxis only and $5.5 \%$ were not prescribed any AC. Patients admitted on full dose AC were excluded from the logistic regression and mortality analysis. AC choices are presented in full in - Supplementary Table $\mathbf{S 2}$ (available in the online version). Warfarin, rivaroxaban, argatroban, and bivalirudin had few patients; these were grouped and classified as "other" and excluded from the logistic regression and mortality analyses.

Mortality outcome was stratified by D-dimer level. Unadjusted Kaplan-Meier survival analysis by first D-dimer level category for those patients not on clinical therapy trials
Table 1 Characteristics of the study population

\begin{tabular}{|c|c|c|}
\hline & $n$ & $\%$ \\
\hline \multicolumn{3}{|c|}{ Race $(n=3,625)$} \\
\hline Black & 1,359 & $37.5 \%$ \\
\hline White & 347 & $9.6 \%$ \\
\hline Other & 1,919 & $52.9 \%$ \\
\hline \multicolumn{3}{|c|}{ Ethnicity $(n=3,625)$} \\
\hline Hispanic & 1,361 & $37.5 \%$ \\
\hline \multicolumn{3}{|c|}{ Age, y $(n=3,625)$} \\
\hline$<50$ & 708 & $19.5 \%$ \\
\hline $50-60$ & 671 & $18.5 \%$ \\
\hline $60-70$ & 836 & $23.1 \%$ \\
\hline $70-80$ & 820 & $22.6 \%$ \\
\hline$>80$ & 590 & $16.3 \%$ \\
\hline \multicolumn{3}{|c|}{ Gender $(n=3,625)$} \\
\hline $\mathrm{F}$ & 1,719 & $47.4 \%$ \\
\hline M & 1,905 & $52.6 \%$ \\
\hline Not given & 1 & $0.0 \%$ \\
\hline \multicolumn{3}{|c|}{ Survival status $(n=3,625)$} \\
\hline Survived & 2,832 & $78.1 \%$ \\
\hline Died $>48 \mathrm{~h}$ & 793 & $21.9 \%$ \\
\hline \multicolumn{3}{|c|}{ eGFR, $\mathrm{mL} / \min (n=3,538)$} \\
\hline $30-120$ & 2,778 & $78.5 \%$ \\
\hline $15-30$ & 366 & $10.3 \%$ \\
\hline $0-15$ & 394 & $11.1 \%$ \\
\hline \multicolumn{3}{|c|}{ D-dimer, $\mu \mathrm{g} / \mathrm{mL}$ (initial $48 \mathrm{~h}, n=2,527$ ) } \\
\hline$<1$ & 779 & $30.8 \%$ \\
\hline $1-<3$ & 1,013 & $40.1 \%$ \\
\hline $3-<10$ & 457 & $18.1 \%$ \\
\hline$\geq 10$ & 278 & $11.0 \%$ \\
\hline \multicolumn{3}{|c|}{ Oxygen saturation (\%) by pulse oximetry $(n=3,599)$} \\
\hline $95 \%$ & 2,127 & $59.1 \%$ \\
\hline $90-95 \%$ & 875 & $24.3 \%$ \\
\hline$<90 \%$ & 597 & $16.6 \%$ \\
\hline \multicolumn{3}{|c|}{ Ventilator required initial $48 \mathrm{~h}(n=3,625)$} \\
\hline No & 3,312 & $91.4 \%$ \\
\hline Yes & 313 & $8.6 \%$ \\
\hline \multicolumn{3}{|c|}{ ICU admission required initial $48 \mathrm{~h}(n=3,625)$} \\
\hline No & 3,373 & $93.1 \%$ \\
\hline Yes & 252 & $7.0 \%$ \\
\hline
\end{tabular}

Abbreviations: eGFR, estimated glomerular filtration rate; F, female; $\mathrm{ICU}$, intensive care unit; $\mathrm{M}$, male.

confirmed the positive association of D-dimer level with mortality (-Supplementary Fig. $\mathbf{5 3}$, available in the online version; $p<0.0001$ ) and its value as a surrogate marker of severity. 


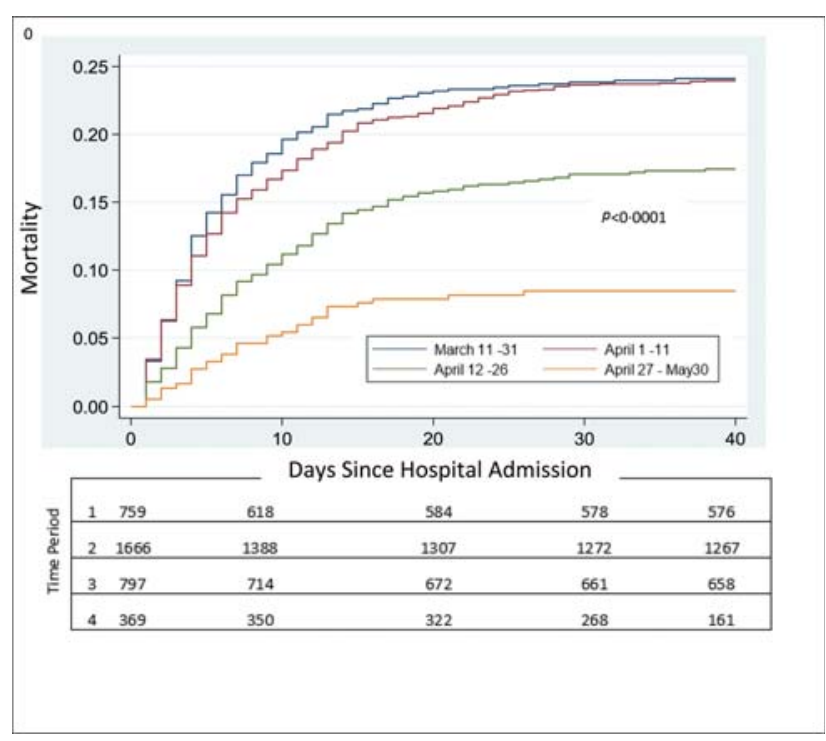

Fig. 1 Cumulative mortality according to time period on presentation.

\section{Chosen Therapy Stratified by D-Dimer Level}

We examined separately these therapies according to D-dimer level. Most patients had D-dimer levels drawn (69.7\%) within 48 hours of admission. The distribution of D-dimer values and the chosen patient therapy are shown in - Supplementary Table $\mathbf{S 3}$ (available in the online version). Choice of AC varied within the D-dimer levels. Patient on no AC were seen throughout the range of D-dimer levels. Of patients with a D-dimer level of $<1 \mu \mathrm{g} / \mathrm{mL}, 74$ (9.6\%) were placed on therapeutic AC while 105 (13.5\%) received no thromboprophylaxis. At a D-dimer level between 1 and $<3 \mu \mathrm{g} / \mathrm{mL}, 162$ (16.0\%) received full therapeutic doses and $116(11.5 \%)$ received no therapy. At a D-dimer value of $\geq 10 \mu \mathrm{g} / \mathrm{mL}, 38(13.7 \%)$ received no thromboprophylaxis or therapy (-Supplementary Table S3, available in the online version).

Comparisons of efficacy of therapy regimens: Adjusted analysis: A logistic model for discharged patients is provided in - Table $\mathbf{2}$ describing the impact of age, oxygen saturation, eGFR, D-dimer level, time period (wave), ICU admission, and AC medicine order and type, all as determined in the first 48 hours of admission on the odds of death $(n=2,450)$. BMI categorically defined as $<30,30$ to 35 , and $>35 \mathrm{~kg} / \mathrm{m}^{2}$ was not significant and was not included in the table. All other parameters were significant and were adjusted for. As expected, age, oxygen saturation $<90 \%$, decreased GFR, elevated D-dimer levels, and ICU admission were all significantly associated with increased mortality. The later time periods, collapsed into two levels and designated as time waves, were also significantly associated with a better prognosis.

When compared with no AC at baseline and after adjustment for the variables above, apixaban prophylaxis (odds ratio [OR] 0.46, $p=0.001$ ), apixaban therapy (OR $0.57, p=0.006$ ), and enoxaparin prophylaxis (OR $0.49, p=0.001$ ) were all associated with a significant decrease in mortality, as shown in -Table 2. UFH was not associated with significant benefit, either as prophylaxis or therapy.
Table 2 Logistic regression analysis of variables: adjusted odds ratios for mortality outcome $(n=2,450)$

\begin{tabular}{|c|c|c|c|}
\hline Variable & $\begin{array}{l}\text { Odds } \\
\text { ratio }\end{array}$ & $\begin{array}{l}95 \% \\
\text { Confidence } \\
\text { interval }\end{array}$ & $p$-Value \\
\hline \multicolumn{4}{|l|}{ Age, y } \\
\hline$<50$ & 1 & & \\
\hline $50-60$ & 2.71 & $1.57-4.71$ & $<0.001$ \\
\hline $60-70$ & 4.9 & $2.43-6.91$ & $<0.001$ \\
\hline $70-80$ & 5.85 & $3.45-9.92$ & $<0.001$ \\
\hline$\geq 80$ & 8.59 & $5.01-14.74$ & $<0.001$ \\
\hline \multicolumn{4}{|l|}{ Oxygen saturation, \% } \\
\hline$>95 \%$ & 1 & & \\
\hline $90-95 \%$ & 1.56 & $1.18-2.05$ & 0.002 \\
\hline$<90 \%$ & 3.25 & $2.45-4.28$ & $<0.001$ \\
\hline \multicolumn{4}{|l|}{ eGFR $\mathrm{mL} / \mathrm{min}$} \\
\hline$>60$ & 1 & & \\
\hline$\geq 30-60$ & 1.76 & $1.33-2.33$ & $<0.001$ \\
\hline $15-30$ & 2.61 & $1.84-3.69$ & $<0.001$ \\
\hline $0-15$ & 1.85 & $1.28-2.67$ & $<0.001$ \\
\hline \multicolumn{4}{|l|}{ D-dimer, $\mu \mathrm{g} / \mathrm{mL}$} \\
\hline$<1$ & 1 & & \\
\hline $1-3$ & 1.81 & $1.30-2.52$ & $<0.001$ \\
\hline $3-10$ & 2.24 & $1.54-3.27$ & $<0.001$ \\
\hline$\geq 10$ & 3.46 & $2.31-5.19$ & $<0.001$ \\
\hline \multicolumn{4}{|l|}{ Time period } \\
\hline First time period & 1 & & \\
\hline Wave & 0.53 & $0.41-0.68$ & $<0.001$ \\
\hline \multicolumn{4}{|l|}{ ICU } \\
\hline No ICU & 1 & & \\
\hline ICU & 3.72 & $2.50-5.52$ & $<0.001$ \\
\hline \multicolumn{4}{|c|}{ Medication and regimen, intent type } \\
\hline No anticoagulation & 1 & & \\
\hline $\begin{array}{l}\text { Apixaban } \\
\text { prophylaxis }\end{array}$ & 0.46 & $0.30-0.71$ & 0.001 \\
\hline $\begin{array}{l}\text { Apixaban full } \\
\text { therapy }\end{array}$ & 0.57 & $0.38-0.85$ & 0.006 \\
\hline $\begin{array}{l}\text { Enoxaparin } \\
\text { prophylaxis }\end{array}$ & 0.49 & $0.32-0.73$ & 0.001 \\
\hline $\begin{array}{l}\text { Enoxaparin full } \\
\text { therapy }\end{array}$ & 0.83 & $0.44-1.56$ & 0.57 \\
\hline $\begin{array}{l}\text { UFH prophylaxis } \\
\text { twice daily }\end{array}$ & 0.79 & $0.54-1.17$ & 0.24 \\
\hline $\begin{array}{l}\text { UFH prophylaxis } \\
\text { thrice daily }\end{array}$ & 1.04 & $0.54-1.17$ & 0.91 \\
\hline $\begin{array}{l}\text { UFH full } \\
\text { therapy }\end{array}$ & 0.97 & $0.51-1.84$ & 0.93 \\
\hline
\end{tabular}

Abbreviations: eGFR, estimated glomerular filtration rate; ICU, intensive care unit; UFH, unfractionated heparin. 
Table 3 Adjusted mortality per therapeutic modality, stratified by D-dimer (DD): odds ratios and confidence intervals ( $n=2,450)$

\begin{tabular}{|c|c|c|c|c|c|c|c|}
\hline \multirow[t]{2}{*}{$n=2,450$} & Apixaban & Apixaban & Enoxaparin & Enoxaparin & Heparin & $\begin{array}{l}\text { Heparin high } \\
\text { dose }\end{array}$ & Heparin \\
\hline & Prophylaxis & Therapy & Prophylaxis & Therapy & Prophylaxis & Prophylaxis & Therapy \\
\hline \multirow{3}{*}{$\begin{array}{l}\mathrm{DD}<1 \\
(n=751)\end{array}$} & 0.85 & 1.76 & 0.86 & 5.85 & 2.21 & 1.35 & 2.51 \\
\hline & $\begin{array}{l}95 \% \mathrm{Cl}: \\
0.26-2.84\end{array}$ & $\begin{array}{l}95 \% \mathrm{Cl}: \\
0.53-5.90\end{array}$ & $\begin{array}{l}95 \% \mathrm{Cl}: \\
0.28-2.61\end{array}$ & $\begin{array}{l}95 \% \text { Cl: } \\
0.49-69.21\end{array}$ & $\begin{array}{l}95 \% \mathrm{Cl}: \\
0.76-6.40\end{array}$ & $\begin{array}{l}95 \% \text { Cl: } \\
0.21-8.64\end{array}$ & $\begin{array}{l}95 \% \text { Cl: } \\
0.32-19.79\end{array}$ \\
\hline & $p=0.80$ & $p=0.36$ & $p=0.79$ & $p=0.16$ & $p=0.14$ & $p=0.75$ & $p=0.38$ \\
\hline \multirow{3}{*}{$\begin{array}{l}\text { DD } 1-<3 \\
(n=991)\end{array}$} & 0.44 & 0.48 & 0.50 & 1.05 & 0.64 & 1.11 & 0.50 \\
\hline & $\begin{array}{l}95 \% \mathrm{Cl}: \\
0.23-0.83\end{array}$ & $\begin{array}{l}95 \% \text { Cl: } \\
0.24-0.97\end{array}$ & $\begin{array}{l}95 \% \text { Cl: } \\
0.27-0.94\end{array}$ & $\begin{array}{l}95 \% \mathrm{Cl}: \\
0.31-3.53\end{array}$ & $\begin{array}{l}95 \% \mathrm{Cl}: \\
0.35-1.18\end{array}$ & $\begin{array}{l}95 \% \mathrm{Cl}: \\
0.45-2.71\end{array}$ & $\begin{array}{l}95 \% \mathrm{Cl}: \\
0.14-1.86\end{array}$ \\
\hline & $p=0.012$ & $p=0.041$ & $p=0.031$ & $p=0.93$ & $p=0.15$ & $p=0.82$ & $p=0.30$ \\
\hline \multirow{3}{*}{$\begin{array}{l}\text { DD } 3-<10 \\
(n=439)\end{array}$} & 0.35 & 0.67 & 0.47 & 0.7 & 0.73 & 0.89 & 0.64 \\
\hline & $\begin{array}{l}95 \% \mathrm{Cl}: \\
0.0 .95-1.30\end{array}$ & $\begin{array}{l}95 \% \mathrm{Cl}: \\
0.32-1.41\end{array}$ & $\begin{array}{l}95 \% \text { Cl: } \\
0.19-1.18\end{array}$ & $\begin{array}{l}95 \% \mathrm{Cl}: \\
0.21-2.34\end{array}$ & $\begin{array}{l}95 \% \mathrm{Cl}: \\
0.30-1.79\end{array}$ & $\begin{array}{l}95 \% \mathrm{Cl}: \\
0.26-3.06\end{array}$ & $\begin{array}{l}95 \% \mathrm{Cl}: \\
0.16-2.57\end{array}$ \\
\hline & 0.12 & $p=0.29$ & $p=0.11$ & $p=0.56$ & $p=0.49$ & $p=0.85$ & $p=0.53$ \\
\hline \multirow{3}{*}{$\begin{array}{l}\text { DD } \geq 10 \\
(n=269)\end{array}$} & 0.26 & 0.22 & 0.13 & 0.57 & 0.63 & 1.55 & 1.06 \\
\hline & $\begin{array}{l}95 \% \mathrm{Cl}: \\
0.068-0.98\end{array}$ & $\begin{array}{l}95 \% \mathrm{Cl}: \\
0.084-0.55\end{array}$ & $\begin{array}{l}95 \% \mathrm{Cl}: \\
0.034-0.49\end{array}$ & $\begin{array}{l}95 \% \mathrm{Cl}: \\
0.18-1.81\end{array}$ & $\begin{array}{l}95 \% \mathrm{Cl}: \\
0.24-1.63\end{array}$ & $\begin{array}{l}95 \% \mathrm{Cl}: \\
0.13-17.83\end{array}$ & $\begin{array}{l}95 \% \text { Cl: } \\
0.34-3.32\end{array}$ \\
\hline & $p=0.047$ & $p=0.001$ & $p=0.003$ & $p=0.34$ & $p=0.34$ & 0.73 & 0.92 \\
\hline
\end{tabular}

Abbreviation: $\mathrm{Cl}$, confidence interval.

We then compared therapy for patients to a baseline of no AC stratified, rather than adjusted, by D-dimer values with adjustment for the other variables in stratum-specific logistic regression models ( - Table 3 ). For D-dimer levels $<1 \mu \mathrm{g} / \mathrm{mL}$, there was no benefit associated with any of the treatments. At D-dimer levels 1 to $<3 \mu \mathrm{g} / \mathrm{mL}$, apixaban at both the prophylactic (OR $0.4495 \%$ confidence interval [CI]: 0.23-0.83, $p=0.012$ ) and therapeutic (OR: 0.48, 95\% CI: 0.24-0.97, $p=0.041$ ) dosing was associated with a significant decrease in mortality ORs as was enoxaparin prophylaxis (OR 0.5, 95\% CI: $0.27-0.94, p=0.031)$. Enoxaparin therapy and UFH at any dosing were not associated with decreased mortality. At D-dimer levels $>10 \mu \mathrm{g} / \mathrm{mL}$, the same pattern held; enoxaparin prophylaxis and apixaban prophylaxis and therapy were all associated with decreased mortality (OR 0.13, 95\% CI: $0.034-$ $0.49, p=0.003$; OR 0.26, 95\% CI: 0.068-0.98, $p=0.047$; and OR $0.22,95 \%$ CI: $0.084-0.55, p=0.001$, respectively), while UFH and enoxaparin therapy were not.

\section{Transfusion Requirement}

We were concerned about the possibility that certain $\mathrm{AC}$ choices, particularly intravenous heparin, might have been influenced by concern over increased bleeding. We reexamined our cohort for those patients requiring transfusion support that were contemporaneous with the anticoagulant order. For the transfusion risk analysis, we eliminated 106 patients who received a transfusion within the first 72 hours of admission since those who actually received a transfusion within the first 72 hours might have been suspected of requiring it at the 48-hour mark when the $\mathrm{AC}$ was chosen and therefore biasing the selection. Note that 614 patients had a length of stay greater than 48 hours but less than 72 hours and were excluded from evaluation. A total of 183 patients did not have a transfusion in the first 3 days but did have a transfusion from day 3 onwards. Logistic regression adjusted models, with same adjusters as in the mortality analysis, did not find any anticoagulant regimen with a likelihood of transfusion greater than for patients on no anticoagulant regimen (-Table 4).

\section{Discussion}

Early in the course of COVID-19, the Shanghai Clinical Treatment Expert Group had suggested that AC may be of some benefit in COVID-19 patients. ${ }^{7}$ Tang et al found no difference in 28-day mortality for the general population of heparin users and nonusers overall, but in patients with sepsis-induced coagulopathy score $\geq 4$ and those patients

Table 4 Logistic regression for transfusion requirement by treatment choice

\begin{tabular}{|l|l|l|l|}
\hline \multicolumn{4}{|l|}{ Bleeding as defined by transfusion requirement } \\
\hline & OR & $95 \% \mathrm{Cl}$ & p-Value \\
\hline None & 1.00 & & \\
\hline Apixaban prophylaxis & 0.68 & $0.31-1.50$ & 0.34 \\
\hline Apixaban full therapy & 0.79 & $0.40-1.57$ & 0.51 \\
\hline Enoxaparin prophylaxis & 0.68 & $0.32-1.44$ & 0.31 \\
\hline Enoxaparin full therapy & 1.31 & $0.51-3.36$ & 0.58 \\
\hline UFH standard prophylaxis & 0.87 & $0.43-1.74$ & 0.69 \\
\hline UFH high prophylaxis & 0.69 & $0.26-1.79$ & 0.45 \\
\hline UFH full therapy & 1.01 & $0.40-2.53$ & 0.99 \\
\hline
\end{tabular}

Abbreviations: $\mathrm{Cl}$, confidence interval; OR, odds ratio; UFH, unfractionated heparin. 
on heparin for $\geq 7$ days had a lower mortality than those not on AC (40.0\% vs. $64.2 \%, p=0.029)$. This benefit was particularly seen in those with the highest D-dimer values and those with $>$ sixfold of upper limit of normal (32.8\% vs. $52.4 \%, p=0.017$ ). Although the study was done at the early onset of COVID-19 and did not analyze for other variables, nor did it differentiate between prophylactic and therapeutic dosing intensity, there was some evidence that AC might be of benefit for some patients. Llitjos et al showed no difference in death between prophylactic (one of eight patients) and therapeutic ( 2 deaths in 18 patients) AC but the small numbers precluded any meaningful conclusion. ${ }^{12}$ Thachil has also suggested that perhaps higher than prophylactic doses should be used in patients with extremely high D-dimer values. ${ }^{13}$

Since data have shown a benefit of DOACs in prophylaxis of high-risk cancer patients, for medically ill patients with acute infectious diseases, and for patients undergoing orthopaedic procedures, we also offered oral AC with a DOAC in our protocol as a way of minimizing direct nursing/patient exposure. ${ }^{14-17}$ Apixaban was chosen as the preferred oral DOAC at our institution for its better safety profile in patients with renal failure, a not uncommon complication of COVID-19+. The NIH ACTIV-4 trial has recently chosen apixaban for its COVID outpatient AC trial. ${ }^{18}$ Our data appear to show that apixaban is associated with approximately the same decrease in OR and improvement in survival as prophylactic enoxaparin, whether this is because of a similar AC effect or a similar anti-inflammatory effect is unknown at this point. ${ }^{19,20}$

The strengths of this article are its large size and mature adjusted determination of baseline risk, establishing relevant variables and therapeutic intent in the first 48 hours. In addition, specific attention to medication type and dose adjusted to patient weight and creatinine clearance allowed us to establish AC therapeutic intent (prophylaxis or full therapy) prior to evaluating the impact of that intent on downstream mortality. The strong association of D-dimer with mortality, as noted in - Supplementary Fig. $\mathbf{5 3}$ (available in the online version), allowed for adjustment of therapy for morbidity and mortality as denoted by D-dimer level.

We were able to examine the value of different regimens and types of AC in COVID-19+ patients. Accepting AC intent as the last anticoagulant order chosen during the first 48hour window after admission, we establish that $A C$ is of benefit in decreasing the ORs for mortality in this disease. Establishing intent prior to a search for outcome alleviates problems often seen in other studies where therapy choice is confounded with outcome. AC begun later in the hospital stay may have been chosen as a response to patient deterioration but may be misinterpreted as being associated with patient deterioration. ${ }^{9}$ We sought to use a cohort, intentionto-treat-like model to preclude confounding by indication and falsely attribute bad outcome to "innocent therapy" added at the time of disease progression.

An additional advantage of this model is that it obviates the "longevity" bias where the longer one survives in the hospital, the greater the chance the clinician might implement AC. Our careful attention to baseline intent obviates both biases and is a major strength of this analysis.

Our very large numbers and our long follow-up, for this disease, allowed us to have a large number with actual outcomes, rather than many studies where, although the initial numbers appear high, many patients are still hospitalized, leaving a question as to what happens to these patients. Only $22(0.5 \%)$ patients of the initial cohort had not been discharged by the censor date. The exclusion of 160 (3.8\%) patients who died within the first 48 hours, necessary for our intent to treat construct, had the additional benefit of excluding immediately terminal patients who might not have lived long enough to gain a potential benefit from AC.

Implementation of the best practice AC protocol was confounded by an improved outcome over time, as has been noted previously in other studies..$^{21,22}$ It could be argued that the improved outcome over time was secondary to reduced admissions in the later time period, improved staffing, and increased experience in managing these critically ill patients but may also have been associated with the acceptance of our AC protocol. To aggressively control for this potential bias, we included, in our logistic modeling, an adjustment for time period. It could be argued that this adjustment might hide the value of AC but, despite this adjustment, the association of AC with survival remained significant, both statistically and clinically. We also eliminated from consideration all patients on a clinical trial whether given therapy or placebo, reducing our sample size by 252 patients (5.95\%), but eliminating the possibility that some of the observed improvement might have been due to the experimental interventions.

We adjusted for severity of illness both in the major logistic regression model and in the stratified model. Despite adjusting for ICU admission, GFR, age, oxygen saturation, time, and Ddimer level, AC with apixaban and with prophylactic enoxaparin were significantly associated with decreased OR for mortality. It is interesting that the reduction in mortality is strikingly similar for the effective therapies throughout the analyses. When stratified by D-dimer values, it appeared that there was no real benefit to any AC at the lowest D-dimer level $<1 \mu \mathrm{g} / \mathrm{mL}$, but as the D-dimer values increased, the ORs with apixaban and enoxaparin prophylaxis were further decreased.

\section{Limitations}

With all our care, this is still an observational cohort study with potential confounders that we may not yet have adequately addressed, but even in prospective studies, there may be confounders of which we are not initially aware. We cannot with certainty eliminate the possibility that certain clinicians who supervised care in the most acute intensive care areas did not have a predilection for one of the therapies, which could have created an artificial relationship between severity and therapy choice. Since at the height of COVID-19, we had some intubated patients on the floor who were not in ICUs, these analyses were performed for ventilator requirement with or without ICU 


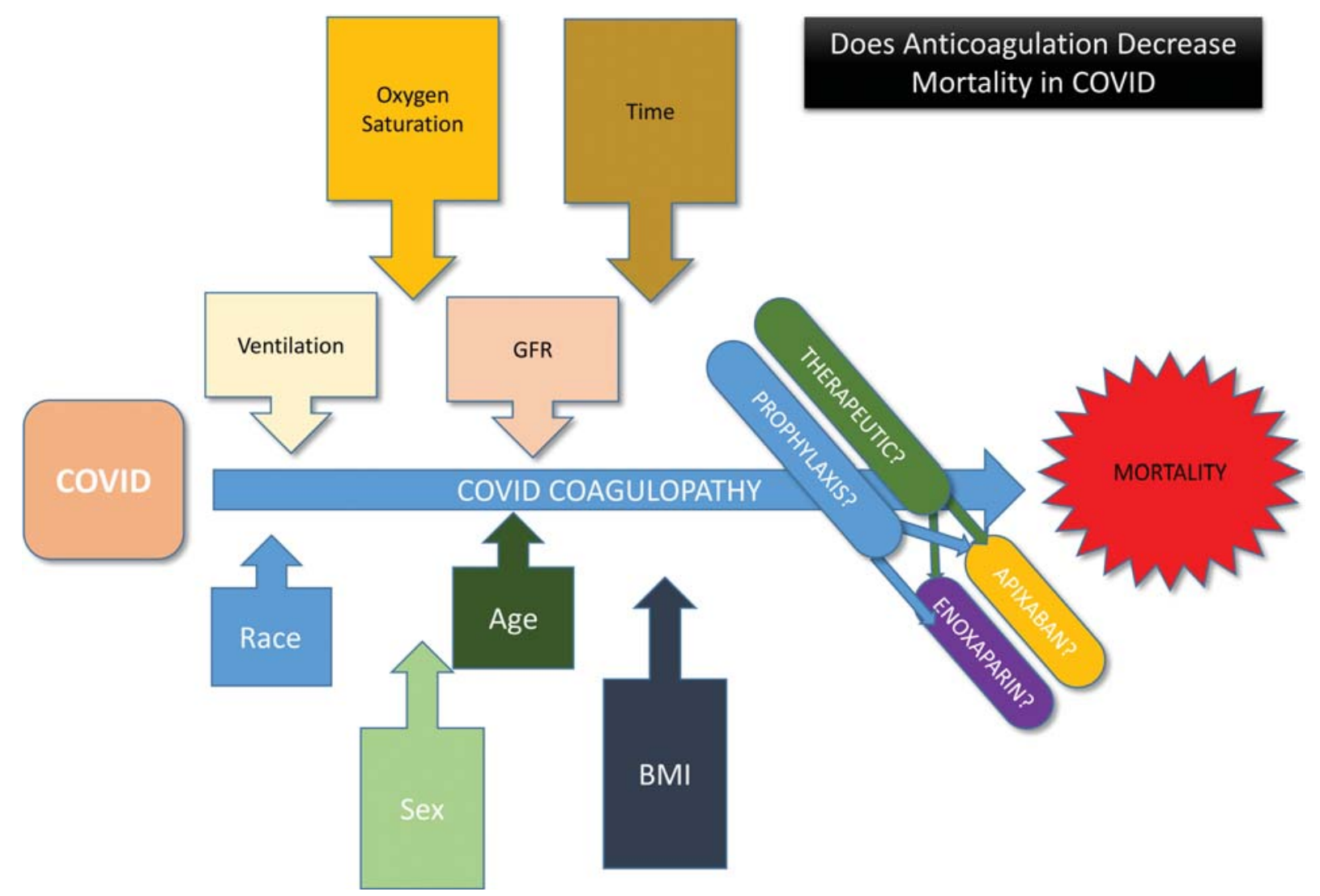

Fig. 2 Visual Summary: Does anticoagulation decrease mortality in coronavirus disease.

status in the first 48 hours; the results were similar (-Supplementary Tables S4 and S5, available in the online version). We did not track how long individual patients received the ordered $A C$ or whether $A C$ choice was switched after the 48-hour time point. We followed an intention-totreat analytic strategy, avoiding the inevitable concern about confounding by indication if we had considered "posttherapy assignment" medication changes. Although we used transfusion as a surrogate for significant bleeding, we understand that this does not take into account intracranial or critical site bleeds that would not necessarily entail transfusion support. We did not examine the benefit of the different types of AC regimens for thrombosis prevention, eventual ventilator requirement, eventual need for dialysis, and ICU admission, nor the relationship of thrombosis to mortality. This is the subject of future analyses because of the intense medical record and image reviews required to ensure data accuracy.

No other published studies have examined apixaban in hospitalized COVID- 19 patients. An oral drug with low bleeding risk and limited renal clearance is advantageous in a disease frequently complicated by acute kidney injury, kidney failure, and dialysis. Reducing nursing contact time by eliminating subcutaneous and intravenous drug administration protects against staff disease transmission.

We report here a very large cohort study which shows a survival benefit, after adjustment for age, ventilation re-
quirement/ICU admission, oxygen saturation, and time period, with AC for patients with COVID-19. We conclude that COVID-19+ patients with moderate or severe illness benefit from $A C$ and that apixaban has similar efficacy to the heparins in decreasing mortality in this disease. Although benefit with therapeutic levels of AC with apixaban was also seen, therapeutic dosing offered no further benefit seen over thromboprophylaxis.

There is great debate whether AC is necessary for COVID$19+$ patients and whether there is anything to be gained by using higher doses, particularly in sicker patients (-Fig. 2). Currently, most guidelines recommend prophylaxis but do not recommend therapeutic AC in the absence of documented thrombi, ${ }^{23,24}$ although the data to support these recommendations are still lacking. Our study demonstrates that AC improves survival in COVID-19+ patients but does not support a preference for higher, therapeutic doses in the COVID-19+ population, even for those who have severe disease.

There are prospective randomized, controlled, multicenter studies underway, like the ACTIV-4 Inpatient and the ATTACC trials, that will determine whether there is any advantage to therapeutic AC versus prophylaxis. Until such time as these randomized controlled studies are complete, it would seem appropriate to treat hospitalized COVID-19+ patients with D-dimer levels $>1 \mu \mathrm{g} / \mathrm{mL}$ with enoxaparin and apixaban prophylactic AC. 


\section{What is known about this topic?}

- COVID-19 is recognized as a prothrombotic disease with a high mortality in hospitalized patients. Anticoagulation has been advocated by many guidelines as potentially beneficial in reducing mortality. The evidence to support this, however, has been sparse.

- It is unclear whether therapeutic anticoagulation, particularly in those with severe disease, is more beneficial than thromboprophylaxis.

\section{What does this paper add?}

- In a large cohort of 3,625 hospitalized COVID-19 patients with known outcomes, we analyzed the results of anticoagulation with therapeutic intent in the first 48 hours of hospitalization on downstream mortality in an observational intention-to-treat analysis.

- We examined the use of both parenteral and oral medication and both prophylactic and therapeutic levels. We adjusted for age, renal function, oxygen saturation, ventilation status, ICU admission, race, time period, gender, and severity of illness as defined by D-dimer levels.

- Our data show that anticoagulation was associated with decreased mortality in patients with COVID-19. Therapeutic anticoagulation does not offer any increased benefit over prophylactic anticoagulation in patients with COVID-19, even in patients with high Ddimer levels.

\section{Note}

For original data, please contact the corresponding author.

\section{Authors' Contributions}

H.H.B. originated the concept, helped in the analysis, and wrote the manuscript. M.R.G. helped originate the concept, helped in the analysis, and reviewed and edited the manuscript. J.S. helped in the statistical analysis and reviewed and edited the manuscript. K.I. helped in the analysis and reviewed and edited the manuscript. L.S. developed the cohorts, helped in the statistical analysis, and reviewed and edited the manuscript. Y.L. helped in the statistical analysis and reviewed and edited the manuscript. S.R. reviewed patient data and reviewed and edited the manuscript. J.D.G.L. reviewed patient data and reviewed and edited the manuscript. M.K. reviewed the data and edited the manuscript. M.B. reviewed patient data and reviewed and edited the manuscript. L.G. reviewed the data and edited the manuscript. E.B. developed the CLG program, developed the cohorts, performed the statistical analyses, and reviewed and edited the manuscript.
Conflict of Interest

H.H.B. has received research or advisory funding from Bristol Myers Squibb, Janssen Pharmaceuticals, Bayer, Kedrion Pharmaceuticals, and Alexion Pharmaceuticals. M.K. has received research or advisory funding from Bristol Myers Squibb and Janssen Pharmaceuticals. All other authors declare no competing interests.

\section{Acknowledgments}

We greatly appreciate the efforts of our fellow health care workers and support staff at the Montefiore Medical Center for providing outstanding patient care at considerable personal risk on the front lines of this pandemic. We express our support for our patients with COVID-19 and their families.

\section{References}

1 Chen N, Zhou M, Dong X, et al. Epidemiological and clinical characteristics of 99 cases of 2019 novel coronavirus pneumonia in Wuhan, China: a descriptive study. Lancet 2020;395(10223):507-513

2 Middeldorp S, Coppens M, van Haaps TF, et al. Incidence of venous thromboembolism in hospitalized patients with COVID-19. J Thromb Haemost 2020;18(08):1995-2002

3 Lodigiani C, Iapichino G, Carenzo L, et al; Humanitas COVID-19 Task Force. Venous and arterial thromboembolic complications in COVID-19 patients admitted to an academic hospital in Milan, Italy. Thromb Res 2020;191:9-14

4 Helms J, Tacquard C, Severac F, et al; CRICS TRIGGERSEP Group (Clinical Research in Intensive Care and Sepsis Trial Group for Global Evaluation and Research in Sepsis). High risk of thrombosis in patients with severe SARS-CoV-2 infection: a multicenter prospective cohort study. Intensive Care Med 2020;46(06):1089-1098

5 Liu XL, Wang XZ, Liu XX, et al. Low-dose heparin as treatment for early disseminated intravascular coagulation during sepsis: a prospective clinical study. Exp Ther Med 2014;7(03):604-608

6 Connors JM, Levy JH. COVID-19 and its implications for thrombosis and anticoagulation. Blood 2020;135(23):2033-2040

7 Tang N, Bai H, Chen X, Gong J, Li D, Sun Z. Anticoagulant treatment is associated with decreased mortality in severe coronavirus disease 2019 patients with coagulopathy. J Thromb Haemost 2020;18(05):1094-1099

8 Hanif A, Khan S, Mantri N, et al. Thrombotic complications and anticoagulation in COVID-19 pneumonia: a New York City hospital experience. Ann Hematol 2020;99(10):2323-2328

9 Paranjpe I, Fuster V, Lala A, et al. Association of treatment dose anticoagulation with in-hospital survival among hospitalized patients with COVID-19. J Am Coll Cardiol 2020;76(01):122-124

10 Bellin E, Fletcher DD, Geberer N, Islam S, Srivastava N. Democratizing information creation from health care data for quality improvement, research, and education-the Montefiore Medical Center Experience. Acad Med 2010;85(08):1362-1368

11 Bellin E. Missing Management: Health-Care Analytic Discovery in a Learning Health System. South Carolina: Kindle Direct Publishing; 2019

12 Llitjos JF, Leclerc M, Chochois C, et al. High incidence of venous thromboembolic events in anticoagulated severe COVID-19 patients. J Thromb Haemost 2020;18(07):1743-1746

13 Thachil J. The versatile heparin in COVID-19. J Thromb Haemost 2020;18(05):1020-1022

14 Cohoon KP, De Sanctis Y, Haskell L, McBane RD, Spiro TE. Rivaroxaban for thromboprophylaxis among patients recently hospitalized for acute infectious diseases: a subgroup analysis of the MAGELLAN study. J Thromb Haemost 2018;16(07): $1278-1287$ 
15 Khorana AA, Soff GA, Kakkar AK, et al; CASSINI Investigators. Rivaroxaban for thromboprophylaxis in high-risk ambulatory patients with cancer. N Engl J Med 2019;380(08):720-728

16 Yoshida RdeA, Yoshida WB, Maffei FH, El Dib R, Nunes R, Rollo HA. Systematic review of randomized controlled trials of new anticoagulants for venous thromboembolism prophylaxis in major orthopedic surgeries, compared with enoxaparin. Ann Vasc Surg 2013;27(03):355-369

17 Carrier M, Abou-Nassar K, Mallick R, et al; AVERT Investigators. Apixaban to prevent venous thromboembolism in patients with cancer. N Engl J Med 2019;380(08):711-719

18 NIH ACTIV initiative launches adaptive clinical trials of bloodclotting treatments for COVID-19. Available at: https://www.nih. gov/news-events/news-releases/nih-activ-initiative-launchesadaptive-clinical-trials-blood-clotting-treatments-covid-19. Accessed October 12, 2020

19 Papadaki S, Tselepis AD. Nonhemostatic activities of factor Xa: are there pleiotropic effects of anti-FXa direct oral anticoagulants? Angiology 2019;70(10):896-907
20 Al-Harbi NO, Imam F, Alharbi MM, et al. Role of rivaroxaban in sunitinib-induced renal injuries via inhibition of oxidative stressinduced apoptosis and inflammation through the tissue necrosis factor- $\alpha$ induced nuclear factor-карра B signaling pathway in rats. J Thromb Thrombolysis 2020;50(02):361-370

21 Petrilli CM, Jones SA, Yang J, et al. Factors associated with hospital admission and critical illness among 5279 people with coronavirus disease 2019 in New York City: prospective cohort study. BMJ 2020;369:m1966

22 Wang M, Zhang J, Ye D, et al. Time-dependent changes in the clinical characteristics and prognosis of hospitalized COVID-19 patients in Wuhan, China: a retrospective study. Clin Chim Acta 2020;510:220-227

23 COVID-19 and VTE/Anticoagulation: Frequently Asked Questions. Available at: https://www.hematology.org/covid-19/covid-19and-vte-anticoagulation. Accessed October 12, 2020

24 Thachil J, Juffermans NP, Ranucci M, et al. ISTH DIC subcommittee communication on anticoagulation in COVID-19. J Thromb Haemost 2020;18(09):2138-2144 